\title{
BMJ Open A scoping review protocol to map the research foci trends in tobacco control over the last decade
}

\author{
Gayle Halas, ${ }^{1}$ Annette S H Schultz, ${ }^{2}$ Janet Rothney, ${ }^{3}$ Leah Goertzen, ${ }^{4}$ \\ Pamela Wener, ${ }^{5}$ Alan Katz ${ }^{6}$
}

To cite: Halas G, Schultz ASH, Rothney J, et al. A scoping review protocol to map the research foci trends in tobacco control over the last decade. BMJ Open 2015;5:e006643. doi:10.1136/bmjopen-2014006643

- Prepublication history and additional material is available. To view please visit the journal (http://dx.doi.org/ 10.1136/bmjopen-2014006643).

Received 15 September 2014 Revised 2 December 2014 Accepted 3 December 2014

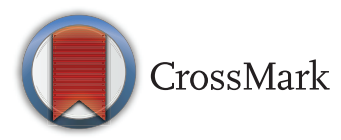

For numbered affiliations see end of article.

\section{Correspondence to}

Alan Katz;

alan_katz@cpe.umanitoba.ca

\section{ABSTRACT}

Introduction: Tobacco dependence and smoke exposure have been global epidemics with health consequences recognised by the US Surgeon General since the 1960s and 1970s, respectively. During this period, a vast body of research evidence has emerged including many reviews of primary research studies targeting various tobacco control strategies. Published review studies synthesise primary evidence, providing a rich source for mapping the broad range of topics and research foci along with revealing areas of evidence deficits. In this paper, we outline our scoping review protocol to systematically review published review articles specific to tobacco control and primary prevention over the last 10 years.

Methods and analysis: Using Arksey and O'Malley's scoping review methodology as a guide, our scoping review of published reviews begins by searching several databases: PubMed, Scopus, the Cochrane Library, the Cumulative Index to Nursing and Allied Health Literature (CINAHL), Psyclnfo and the Educational Resources Information Centre (ERIC). Our multidisciplinary team has formulated search strategies and two reviewers will independently screen eligible studies for final study selection. Bibliographic data and abstract content will be collected and analysed using a tool developed iteratively by the research team.

Ethics and dissemination: A scoping review of published review articles is a novel approach for examining the breadth of literature regarding tobacco control strategies and, as a secondary analysis, does not require ethics approval. We anticipate results will identify research gaps as well as novel ideas for primary prevention research specific to tobacco control strategies concerning intervention, programming and policy. Although this is our first step in establishing a foundation for a research agenda, we will be disseminating results through journals and conferences targeting primary care providers and tobacco control.

In the 1960s, a US Surgeon General report recognised that tobacco use was linked to serious health consequences. ${ }^{1}$ A decade later, another report announced that tobacco smoke exposure was likewise an underlying contributor to what is now considered a long-standing global health epidemic. Since these public acknowledgements, a vast body of research evidence has developed around the health

\section{Strengths and limitations of this study}

- This is a novel review approach to cover a vast volume of literature on a broad topic, thus offering a 'big picture' or map of research on tobacco control within the context of primary prevention.

- A feasible strategy to identify research foci and knowledge gaps within the last 10 years of tobacco control research is presented.

- A practical method to synthesise research that has used a wide range of methodological approaches, settings, study populations and behaviours is described.

- A less detailed analysis of project-specific interventions and research approaches is provided.

- Due to the heterogeneity and breadth of the included studies, the final data extraction framework will not be complete until the review is concluded.

- The synthesis of data will be limited to peerreviewed published work.

consequences of tobacco use and a range of tobacco control strategies. Although improvement is evident, tobacco use remains significant and the costs of treating people with tobacco-related health conditions are astronomical. ${ }^{2}$ As the aim of primary prevention strategies is to prevent illness among the general population, it seems logical that tobacco control strategies are considered within this domain of prevention. Given the large corpus of research literature covering tobacco control and our interests in primary prevention, we describe a protocol for a scoping review of published reviews specific to tobacco control situated within primary prevention.

\section{BACKGROUND}

In developed countries, tobacco use and smoke exposure rates have fallen since the 1960s. However, the WHO currently reports that nearly 6 million premature deaths each year are attributable to either tobacco use or smoke exposure, which is higher than all 
deaths resulting from HIV/AIDS, tuberculosis and malaria together. ${ }^{3}$ Nearly one third to one half of tobacco users will develop a tobacco-related chronic illness leading to death, the most common conditions being cancers, cardiovascular diseases and respiratory ailments. ${ }^{3}$

In response to this ongoing global tobacco epidemic, the WHO developed the Framework Convention on Tobacco Control (FCTC), which was the first evidencebased negotiated treaty within this organisation. ${ }^{5}$ In 2005 , the FCTC was ratified by 168 member states, including Canada. In 13 of the 38 articles of the FCTC, numerous evidence-based strategies for reducing tobacco use and exposure to tobacco smoke are addressed and have been adopted globally by the member states. Notably, the FCTC frames tobacco control strategies within two main areas: reducing the demand for tobacco products and reducing the supply of tobacco products.

The FCTC has now been in place for 9 years. The tobacco control measures endorsed in the Framework are reported to be responsible for dramatic reductions in tobacco use ${ }^{6}$ and for increased investment in tobacco control research and surveillance globally. ${ }^{7}$ While there is ongoing interest in sustaining FCTC strategies, alternate 'end-game' strategies and policies to further reduce the effects of the tobacco epidemic are also being considered. ${ }^{6}$ Several decades of tobacco control strategies have been evaluated along with evidence of the health consequences of tobacco use, but has the reflective step of looking broadly across this vast corpus of research been taken? Since few studies have examined the published tobacco research as a whole, Cohen $e t$ a $l^{8}$ undertook a bibliometric analysis to examine the shift in tobacco research foci between two decades. Our scoping review will contribute a long overdue review of the breadth of research and research foci trends since the introduction of the FCTC, specifically strategies within the domain of primary prevention. This means our focus will be on strategies for intercepting the cause of disease among healthy populations rather than tobacco control strategies targeting populations demonstrating initial signs of disease (secondary prevention) or strategies focusing on the reduction of complications among populations with various stages of incurable conditions (tertiary prevention).${ }^{9}$ We have also chosen to respond to the WHO 2008 challenge to close equity gaps within a generation by proposing use of an equity lens in our analysis. ${ }^{10}$ Our Primary Prevention Research Team is working towards defining a programme of research in primary prevention and here, we present a protocol for a scoping review of reviews covering the published tobacco control literature over the last decade.

\section{METHODS/DESIGN}

We considered the various systematic approaches available for reviewing published literature and chose to undertake a scoping review of published reviews as the best method to map the tobacco control research trends over the last decade. Scoping review methodology is particularly useful for examining a broadly covered topic to comprehensively and systematically map the literature and identify key concepts, theories, evidence, or research gaps. Unlike systematic reviews or meta-analyses, scoping reviews do not narrow the parameters of the review to research trials or require quality assessment. Nonetheless, this type of review is rigorous and methodical in its approach to examining the extent, range and nature of research activity in a particular field ${ }^{11}$ while encompassing both empirical and conceptual research with broadly framed questions. ${ }^{12}$

In designing the protocol for our scoping review of reviews, we drew upon Arksey and O'Malley's ${ }^{11}$ seminal work as well as recent scoping review publications. ${ }^{13}{ }^{14}$ Arksey and O'Malley's scoping review framework outlines a five stage approach with each stage discussed below. Adaptations were driven by an intention to develop a feasible approach for reviewing a vast body of literature.

\section{Stage 1: Identifying the research questions}

Arksey and $\mathrm{O}^{\prime}$ Malley ${ }^{11}$ suggest an iterative process for developing one or more guiding research questions, where each revision is driven by increasing familiarity with the literature. We first realised the need for an iterative process when initial searches primarily resulted in tobacco cessation articles, rather than articles addressing other tobacco control strategies. Our intention to comprehensively examine the tobacco control literature within the domain of primary prevention prompted us to seek a framework encompassing all aspects of tobacco control to guide the search. Turning to the FCTC strategic action terms, we identified five research questions to guide our scoping review of reviews (see table 1). Also, when we reflected on the inclusion of an equity lens, we turned to the PRISMA-Equity 2012 Extension, ${ }^{15}$ which identifies multiple components of equity to be addressed in the review.

\section{Stage 2: Identifying relevant studies}

The aim of scoping reviews is to comprehensively address broad research questions; however, parameters are required to guide the search strategy. At this stage, the team deliberated and decided upon criteria for eligibility, databases to search, and formulated a search strategy and key terms.

\section{Eligibility criteria}

The following inclusion criteria were used to guide the search and will also be used when reviewing articles:

- Published in the English language

- Human subjects

- January 2003-March 2014

- All age groups

- Research that targets the general population and only randomly includes individuals with an illness, disease or condition

- Review articles including: systematic reviews, metaanalyses, meta-syntheses, scoping reviews, narrative 
Table 1 List of research questions and operational definitions

\section{Research questions}

1. Which tobacco control strategies are being addressed in the tobacco control literature?

2. Who are the target populations being addressed in the tobacco control literature?

3. How often is equity addressed in the tobacco control literature and how is equity being integrated into published reviews?

4. What barriers and facilitators to implementing tobacco control strategies are identified in the literature?

5. Is intervention effectiveness evident within the tobacco control literature?

\section{Operational definitions}

Tobacco control strategies based on FCTC(5):

- Price and tax measures

- Protection from exposure

- Regulation of contents and product disclosures

- Packaging/labelling

- Education and awareness

- Advertising, promotion, sponsorship

- Cessation

- Illicit trade

- Sales to minors

Target populations:

- Society

- Government

- Industry

Community

- Healthcare

- Schools

- Workplaces

- Family

- Individual

- Child/youth

Equity categories based on the PRISMA extension: ${ }^{18}$

- Population characteristics including gender, age, ethnicity, socioeconomic status

- Assumptions and rationale related to equity are stated

- Intention to address equity as the focus of review, the research question or within the analysis

- Strategy to address root structural source is stated

Barriers and facilitators:

- As stated by author(s)

Effectiveness:

- Intervention outcomes presented by author(s)

- Authors' suggestions for future research

FCTC, Framework Convention on Tobacco Control.

reviews, rapid reviews, critical reviews and integrative reviews

- Research reviews are limited to developed countries including Canada, USA, Europe, UK, Australia and New Zealand, where rates of smoking, income, standard of living and infrastructures may be comparable and influence response trends to tobacco control strategies.

Explicit exclusion criteria identified are:

- Journal articles that are not rigorous reviews (ie, outside of those defined in the inclusion list), such as book reviews, opinion articles, commentaries or editorial reviews

- Research targeting a population because of a diagnosed illness or disease, or interventions targeting treatment of a specific disease, illness or condition.

\section{Databases}

The following electronic databases were searched: PubMed, Scopus, the Cochrane Library, the Cumulative
Index to Nursing and Allied Health Literature (CINAHL), PsycInfo and the Educational Resources Information Centre (ERIC).

\section{Search strategy}

We drew on the WHO FCTC to operationalise search terms for 'tobacco control strategy', which focuses on demand and supply reduction strategies. ${ }^{5}$ Our research team iteratively developed an extensive list of primary and secondary search terms as well as filtering methods. The primary search terms focused on core tobacco-related terms (ie, tobacco, smoke and nicotine). The secondary search terms included a broader set of keywords such as promotion, prevention and interventions as well as tobacco control strategies based on the FCTC (such as pricing, regulation and packaging). The filtering methods included the date range (within the last 10 years), English, Human and a search string to further narrow the results to review articles. We used the Boolean term 'AND' between 
the core tobacco search strategy and each of the other keywords.

Publication titles from a preliminary search were reviewed to inform refinement of terms in consultation with our team. Refinement of search parameters can be illustrated by the following two examples. First, marijuana use is not a topic that we chose to include, but terms such as 'smoking' did not automatically filter out publications around marijuana use. Using the No Explode option for the main MeSH term 'Smoking' eliminated 'Marijuana Smoking' from search results ("Smoking"[Mesh: NoExp]). Second, we need to be mindful of search terms that produce irrelevant outputs but need to be included as they may otherwise eliminate relevant articles. For example, we found many of the search results targeted a particular disease, such as chronic obstructive pulmonary disease (COPD), and are therefore outside the domain of primary prevention. However, if we use COPD as a search term to filter out such articles, we would miss reviews discussing tobacco use in terms of preventing COPD and thus relevant to primary prevention.

The librarian on our team played a key role in determining and testing appropriate keywords, MESH terms and filters to maximise sensitivity and specificity within the search. She was instrumental in modifying and applying search terms to comply with the various bibliographic databases. The complete and final search strategy for PubMed can be found in online supplementary appendix A; further search strategy details across bibliographic databases are available upon request from the first author. Upon completion, the searches from each database were documented and references were imported into database-specific folders in RefWorks, where duplicates were eliminated.

\section{Stage 3: Study selection}

We designed a two-part study selection process. First, titles will be reviewed by a single reviewer to determine eligibility based on the defined inclusion and exclusion criteria. For example, titles that indicate a target population with an existing medical condition or where the research was carried out in an ineligible country will be removed. At this primary stage of the review, any uncertainty with a title will not eliminate the citation for consideration in the second stage. The second part of the selection process will include two independent reviews of the titles and abstracts using the eligibility criteria. At this point, the two reviewers will also assess the systematic approach reported in the abstract. Given that abstracts commonly contain less specific details, we developed criteria (table 2) to determine the acceptable level of rigour based on a preliminary review of the abstracts and an inductive approach drawing on Gough et als observations of varied review methods. ${ }^{16}{ }^{17}$ A PRISMA Flow diagram ${ }^{18}$ (see online supplementary figure 1.0 in appendix B) will report final numbers once the review is completed.

Where differences arise, the reviewers will consult with a third reviewer to reach consensus. When consensus is not reached, those articles will be included in the review. While scoping review methodology does not specify a process for evaluating study quality, ${ }^{11}$ we will only include abstracts that demonstrate evidence of a systematic approach.

\section{Stage 4: Charting the data}

We will collect and sort key pieces of information from the abstracts of the selected articles. Data to be extracted from the large quantity of published research literature reviews will include some standard information (such as author, year of publication, study objectives) and additional information to examine tobacco control strategies and target populations. Daudt et $a l^{13}$ suggest a trial charting exercise and team consultation to ensure consistency with the questions and purpose. Based on a preliminary exercise, we developed a priori categories

\section{Table 2 Inclusion and exclusion criteria determining acceptable reviews}

\begin{tabular}{ll}
\hline Criteria for inclusion & Criteria for exclusion \\
\hline $\begin{array}{l}\text { At least one of the following minimum criterion required in the } \\
\text { abstract: }\end{array}$ & $\begin{array}{l}\text { A review of literature or documents that were not primary } \\
\text { research, for example, a review of websites or industry } \\
\text { documents } \\
\text { Cataloguing various policies across jurisdictions rather } \\
\text { than reporting on evaluation of a policy }\end{array}$ \\
Number of articles retrieved & An analysis of various methodological approaches \\
Date range & \\
Combined with one of the following criteria: & \\
Search terms used & \\
Literature review-is a term or label to describe the article & \\
rather than reporting as a verb: "to review...." & "compred terms such as "comprehensive review" OR \\
"comprenstematic search"
\end{tabular}


Table 3 Data extraction framework

\begin{tabular}{|c|c|c|}
\hline Bibliometrics & Characteristics of the review & Coding the characteristics \\
\hline $\begin{array}{l}\text { Authors } \\
\text { Title } \\
\text { Source } \\
\text { Year of publication } \\
\text { Country } \\
\text { Language }\end{array}$ & Objective(s) & $\begin{array}{l}\text { Action areas: } \\
\text { Price and tax } \\
\text { Protection } \\
\text { Product regulation } \\
\text { Packaging, labelling } \\
\text { - Education, communication, training } \\
\text { - Advertising and promotion } \\
\text { - Cessation (all programming) } \\
\text { - Illicit trade }\end{array}$ \\
\hline Type of review & $\begin{array}{l}\text { Primary=focus on tobacco control } \\
\text { Multi=addressing multiple risk } \\
\text { behaviours }\end{array}$ & $\begin{array}{l}\text { Equity lens } \\
\text { Specified intention or objective related to equity } \\
\text { Identified target groups or structural influences related } \\
\text { to socio-economic status, gender, race, ethnicity, } \\
\text { religion, age, residence, sexual orientation, disability, } \\
\text { other (specify) }\end{array}$ \\
\hline $\begin{array}{l}\text { Number of included studies } \\
\text { Time frame }\end{array}$ & $\begin{array}{l}\text { Intervention descriptors } \\
\text { Outcome measures: } \\
\text { Process } \\
\text { Impact (including behaviour } \\
\text { change) } \\
\text { Outcome (including health } \\
\text { events/measures) } \\
\text { Target population: } \\
\text { Individual (specify-youth, child, } \\
\text { adult) } \\
\text { Family } \\
\text { Community } \\
\text { Students/school } \\
\text { Employees/workplace } \\
\text { Healthcare } \\
\text { Society } \\
\text { Effectiveness: } \\
\text { Intervention outcomes presented } \\
\text { by author(s) } \\
\text { Future research directions } \\
\text { offered by author(s) }\end{array}$ & \\
\hline
\end{tabular}

which will guide the extraction and charting of data from the abstracts (table 3). The abstracts provide the data necessary for addressing the main objective of this scoping review of reviews, that is, mapping the action areas and target populations covered in the tobacco control literature. However, additional categories may emerge during the data collection process and we may also find some eligible abstracts with missing data. If additional data extraction categories are needed or if missing data emerge, consultation with our research team will guide decisions and will be reported with the findings.

\section{Stage 5: Collating, summarising and reporting the results}

The unique purpose of a scoping review is to aggregate the findings and present an overview rather than a metasynthesis reporting results on narrowly defined questions. The inherent challenges are in determining a framework for presenting a narrative account. ${ }^{11}$ While this approach is still an iterative work in progress, the WHO FCTC strategies are likely to guide our descriptive and visual presentation of results. Additionally, we will be able to identify gaps in the research targeting specific populations and action areas and determine where more in-depth analysis is required. We propose using the PRISMA reporting guidelines for systematic reviews ${ }^{18}$ including components of equity ${ }^{15}$ to accurately report the review search results and analysis summary.

\section{CONCLUSION}

Our protocol for systematically conducting a scoping review of published review articles specific to tobacco control and primary prevention over the last 10 years has been presented. This scoping review of reviews is a novel approach that offers a feasible means for 
synthesising a wide range of research literature specific to tobacco control strategies within the domain of primary prevention. As this will be a first scoping review of reviews within this topic area, our results will advance the scoping review methodology. Results will provide unique insights concerning the extent and scope of tobacco control research foci useful for research and end-user communities. Against the backdrop of a decade of FCTC strategies, we will identify research foci trends and potential gaps specific to the domain of primary prevention. A reflective analysis of this large corpus of published tobacco control research as a whole may reveal new upstream and downstream directions for tobacco control research to prevent tobacco-related morbidity.

\section{Author affiliations}

${ }^{1}$ Department of Family Medicine, College of Medicine, Faculty of Health Sciences, University of Manitoba, Winnipeg, Manitoba, Canada

${ }^{2}$ College of Nursing, Faculty of Health Sciences, University of Manitoba, Winnipeg, Manitoba, Canada

${ }^{3}$ Neil John Maclean Health Sciences Library, University of Manitoba, Winnipeg, Manitoba, Canada

${ }^{4}$ Faculty of Kinesiology and Recreation Management, Department of Family Medicine, College of Medicine, University of Manitoba, Winnipeg, Manitoba, Canada

${ }^{5}$ Department of Occupational Therapy, Faculty of Health Sciences, College of Rehabilitation Sciences, University of Manitoba, Winnipeg, Manitoba, Canada ${ }^{6}$ Departments of Community Health Sciences and Family Medicine, Faculty of Health Sciences, College of Medicine, University of Manitoba, Winnipeg, Manitoba, Canada

Contributors All authors have made substantive intellectual contributions to the development of this protocol. AK conceptualised the review approach and provided general guidance to the research team. All authors were involved in developing the review questions and the review design. AS identified the framework from which GH and JR developed and tested search terms. LG, AK and AS initially developed the data extraction framework which was then further developed by input from team members. GH and AS initiated the first draft of the manuscript which was then followed by numerous iterations with substantial input and appraisal from all of the authors. All authors approve the final version of the manuscript.

Funding This work was supported by the Manitoba Research Chair in Primary Prevention awarded to AK by the Manitoba Health Research Council and the Heart and Stroke Foundation of Manitoba.

Competing interests None.

Provenance and peer review Not commissioned; externally peer reviewed.

Open Access This is an Open Access article distributed in accordance with the Creative Commons Attribution Non Commercial (CC BY-NC 4.0) license, which permits others to distribute, remix, adapt, build upon this work noncommercially, and license their derivative works on different terms, provided the original work is properly cited and the use is non-commercial. See: http:// creativecommons.org/licenses/by-nc/4.0/

\section{REFERENCES}

1. US Department of Health and Human Services. The health consequences of smoking-50 years of progress: a report of the Surgeon General. Atlanta, GA: U.S. Department of Health and Human Services, Centers for Disease Control and Prevention, National Center for Chronic Disease Prevention and Health Promotion, Office on Smoking and Health, 2014.

2. Fiore $\mathrm{MC}$, Jaén $\mathrm{CR}$, Baker TB, et al. Treating tobacco use and dependence: 2008 update. Clinical practice guideline. Rockville, MD U.S. Department of Health and Human Services. Public Health Service, 2008.

3. World Health Organization. WHO global report: Mortality attributable to tobacco. Geneva: WHO, 2012.

4. Asma S, Song Y, Cohen J, et al. CDC Grand Rounds: Global Tobacco Control Centers for Disease Control and Prevention Morbidity and Mortality Weekly Report. Atlanta: Center for Disease Control, 2014. Report No.: Vol 63, No 13.

5. World Health Organization. WHO Framework Convention on Tobacco Control. Geneva: World Health Organization, 2005.

6. Myers ML. The FCTC's evidence-based policies remain a key to ending the tobacco epidemic. Tob Control 2013;22(Suppl 1):i45-6.

7. Yach D, Pratt A, Glynn TJ, et al. Research to stop tobacco deaths Globalization Health 2014;10:39.

8. Cohen JE, Chaiton MO, Planinac LC. Taking stock: A bibliometric analysis of the focus of tobacco research from the 1980s to the 2000s. Am J Prev Med 2010;39:352-6.

9. Cohen L, Chavez V, Chehimi S. Prevention is primary: strategies for community well-being. 2nd edn. San Francisco: Jossey-Bass, 2010.

10. Canadian Institute for Health Information. Health region interventions that address the social determinants of health: equity and structural lenses in intervention research. Ottawa: Canadian Institute for Health Information, 2013.

11. Arksey H, O'Malley L. Scoping studies: towards a methodological framework. Int J Soc Res Methodol 2005;8:19-32.

12. Grimshaw J. A knowledge synthesis chapter. Ottawa: Canadian Institute of Health Research, 2010.

13. Daudt HML, Van Mossel C, Scott SJ. Enhancing the scoping study methodology: A large, inter-professional team's experience with Arksey and O'Malley's framework. BMC Med Res Methodol 2013:13:48

14. Levac D, Colquhoun H, O'Brien KK. Scoping studies: Advancing the methodology. Implementation Sci 2010;5:69.

15. Welch V, Petticrew M, Tugwell P, et al. PRISMA-equity 2012 extension: Reporting guidelines for systematic reviews with a focus on health equity. PLoS Med 2012;9:e1001333.

16. Gough D, Thomas J, Oliver S. Clarifying differences between review designs and methods. Syst Rev 2012;1:28.

17. Gough D. Meta-narrative and realist reviews: guidance, rules, publication standards and quality appraisal. BMC Med 2013;11:22.

18. Moher D, Liberati A, Tetzlaff J, et al. the PRISMA Group. Preferred reporting items for systematic reviews and meta-analyses: The PRISMA statement. PLoS Med 2009;6:e1000097. 